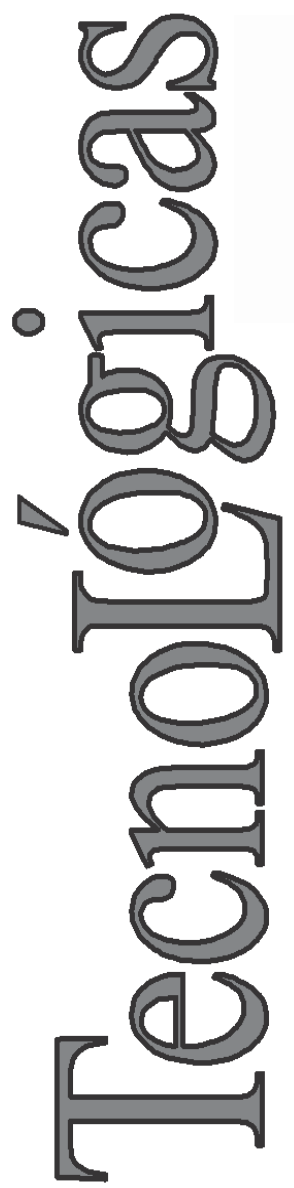

\title{
Módulo Inalámbrico para el Sensado de Vibraciones Superficiales en Suelos
}

\author{
Wireless Module for Sensing \\ Superficial Vibrations of Soils
}

Marlon R. Fulla ${ }^{1}$

Juan L. Palacio-Bedoya ${ }^{2}$

Camilo A. Flórez-Velásquez ${ }^{3}$

Víctor H. Aristizábal-Tique ${ }^{4}$

1 Departamento de Electrónica, Facultad de Ingeniería,

Institución Universitaria Pascual Bravo, Medellín-Colombia mrfulla@pascualbravo.edu.co

2 Departamento de Biología, Facultad de Ciencias y

Biotecnología, Universidad CES, Medellín-Colombia jpalaciob@ces.edu.co

3 Facultad de Telecomunicaciones, Universidad Santo

Tomás, Medellín-Colombia camilo.florez@ustamed.edu.co

4 Facultad de Ingeniería, Universidad Cooperativa de Colombia, Medellín-Colombia vharisti@yahoo.com 


\title{
Resumen
}

En el presente trabajo se evalúa la viabilidad de implementar la tecnología XBee en el desarrollo de sensores acelerométricos inalámbricos (SAI) para el registro en superficie de las vibraciones que generan las ondas sísmicas que se propagan en el suelo. Se verificó experimentalmente la incidencia de la distancia y de la presencia de obstáculos en el radioenlace establecido entre un coordinador y un dispositivo final, mediante la determinación del número de paquetes recibidos exitosamente en diferentes condiciones de operación. Adicionalmente se determinó la influencia de la velocidad de transmisión sobre la frecuencia de muestreo de señales asociadas a vibraciones mecánicas provenientes de un terreno de prueba, a través de la medición de los periodos de muestreos efectivos del proceso "Conversión A/D - Transmisión". Se concluye que los errores en la recepción de los paquetes de datos introducidos por la atenuación del canal y por la presencia de obstáculos, imponen serias restricciones sobre la distancia máxima permisible entre los módulos de comunicación. Las velocidades de transmisión características de la tecnología XBee en asocio con el tiempo de conversión A/D del microcontrolador, permiten llevar a cabo registros a una frecuencia máxima de muestreo de $1 \mathrm{kHz}$; útil para aplicaciones en tiempo real de prospección sísmica donde las señales típicas están dentro un rango espectral de 0 a $500 \mathrm{~Hz}$. Para incrementar la frecuencia de muestreo del sensor para aplicaciones de prospección con señales de anchos de banda superiores a los $500 \mathrm{~Hz}$, se probó exitosamente un prototipo que emplea una memoria externa de rápida escritura para el almacenamiento de datos, mejorando significativamente el muestreo de la señal y que rescata la tecnología XBee debido a sus excelentes características de bajo consumo.

\section{Palabras clave}

Vibraciones superficiales; XBee; punto a punto; Freescale Freedom; Acelerómetro.

\begin{abstract}
In the present work, the feasibility of implementing the XBee technology in wireless accelerometric sensors (WAS) development for sensing of elastic waves on soils surface is analyzed. The incidence of distance and obstacles between a coordinator and end-device pair in their radio link by examining the number of packets received successfully was verified. Additionally, it was investigated the influence of the transmission rate over the sampling frequency of signals associated to mechanical vibrations from a testing ground by measuring the effective sampling periods of the "A / D Conversion - Transmission" process. The data reception errors introduced by the channel attenuation and the presence of obstacles, impose severe restrictions on the maximum allowable distance between the communication modules. The transmission rate features provided by XBee technology in association with the A / D time sampling of the microcontroller, allow to carry out recordings to a maximum sampling frequency of $1 \mathrm{kHz}$, useful for real-time applications where seismic signals are into the spectral range 0 to $500 \mathrm{~Hz}$. In order to increase the sampling frequency of the sensor for prospection applications with signals with bandwidths greater than $500 \mathrm{~Hz}$, it was successfully tested a prototype that uses a fast external memory for storing data, which significantly improves the sampling signal allowing to retake XBee technology due to its excellent low consumption features.
\end{abstract}

\section{Keywords}

Superficial vibrations; XBee; point to point; Freescale Freedom; accelerometer. 


\section{INTRODUCCIÓN}

La inferencia de las propiedades físicas de las capas superficiales de suelos geográficos o de discontinuidades físicas localizadas en estos, como cavidades, objetos de diversos materiales y geometrías o cambios de estas propiedades debido a eventos, continúa siendo materia de intensa investigación. Áreas de la ingeniería como la civil (Ryden \& Mooney, 2009; Sun \& Wenb, 2013), las ciencias forenses y arqueológicas (García-García et al., 2013; Zhao et al., 2013), el sector militar (Scott et al., 2001; Xiang \& Sabatier, 2000; Lou et al., 2013) y el sector de minas y energía (Picozzi et al., 2010; Shu et al., 2012; Savazzi et al., 2013) requieren de desarrollos tecnológicos para este propósito, que cumplan con los requisitos característicos de cada disciplina.

Para llevar a cabo una caracterización del subsuelo desde una perspectiva sísmica, algunos autores (Virieux, 1986; Xiang \& Sabatier, 2000; Ryden \& Mooney, 2009; Watt, 2013; Savazzi et al., 2013) han propuesto el estudio interferométrico de ondas elásticas generadas por fuentes mecánicas en superficie, como electrovibradores, parlantes o explosiones. Estas ondas son inducidas en el suelo y en su proceso de propagación bajo la superficie, interfieren y se difractan al encontrarse una discontinuidad, posteriormente se reflejan de nuevo a la superficie generando como consecuencia un patrón de vibraciones característico que depende fuertemente de las propiedades geométricas y físicas de las discontinuidades del medio.

Kim et al. (2007) implementaron una red de sensores acelerométricos inalámbricos (RSAI) tipo estrella de 64 sensores anclada al célebre puente norteamericano Golden Gate aplicando el estándar IEEE 802.15.4. Esta solución presenta algunas dificultades técnicas importantes: La primera es el rápido desbordamiento de la memoria interna de los dispositivos activos con los datos muestreados debido a la limitada capacidad de la memoria no volátil empleada $(512 \mathrm{kB})$. La segunda dificultad son las restricciones de potencia y velocidad de transmisión, por la necesidad de mantener activos los equipos de la red durante largos periodos de tiempo (autonomía) y por último, la topología elegida de la red impone largos tiempos de recolección de todas las señales de interés del 
puente (12 horas) lo cual impide ejecutar un monitoreo en tiempo real de esa estructura. Este tiempo puede ser reducido utilizando algoritmos de compresión (Xu et al., 2004) con posibles pérdidas de información, que son tolerables en algunas aplicaciones, pero no son aceptables en experimentos donde se requiera capturar señales en el dominio del tiempo con excelente precisión numérica (Scott et al., 2001).

En la presente contribución se expone el diseño e implementación de una RSAI punto a punto cuya finalidad es examinar la viabilidad del uso del estándar IEEE 802.15.4 para aplicaciones en prospección de subsuelos. Los factores tenidos en cuenta en la evaluación de la calidad de la red son: i) el grado de influencia de los obstáculos presentes en el radioenlace y la longitud del canal sobre la confiabilidad, y ii) la frecuencia de muestreo efectiva de la red contra la frecuencia de muestreo requerida para capturar señales análogas provenientes de pruebas sísmicas.

\section{CONFIGURACIÓN DE LA RSAI Y HARDWARE}

La topología seleccionada para la RSAI es del tipo punto a punto, cuya topología permite evaluar de una forma simplificada la influencia de los obstáculos y de la longitud del enlace inalámbrico en la confiabilidad de los paquetes transmitidos. Para implementar la RSAI se seleccionó la tecnología XBee Pro S1 (Villa et al., 2009), debido a que su potencia de transmisión (típicamente $60 \mathrm{~mW}$ ) sirve para establecer radioenlaces de grandes longitudes (hasta $100 \mathrm{~m}$ en interiores y $1500 \mathrm{~m}$ en exteriores), lo que permite mayor cobertura en aplicaciones de prospección sísmica.

\subsection{Descripción de los Nodos Coordinador y Sensor}

El Hardware del nodo sensor (ver Fig. 1) está constituido por una plataforma de desarrollo Freescale Freedom con procesador $\mathrm{ARM}{ }^{\circledR}$ Cortex ${ }^{\mathrm{TM}}-\mathrm{M} 0+$. El nodo registra, empleando conversores de 16 bits, tres señales analógicas provenientes de un acelerómetro triaxial MMA7260QT, previamente acondicionadas (ver Fig. 1 elemento (c)), el cual puede responder adecuadamente a vibracio- 
nes con frecuencias de hasta $1 \mathrm{kHz}$. Empleando un protocolo definido por el usuario, es posible realizar la conversión A/D y la transmisión iterativa de los datos en un paquete de 12 bytes 0 almacenarlos en una tarjeta de memoria no volátil.

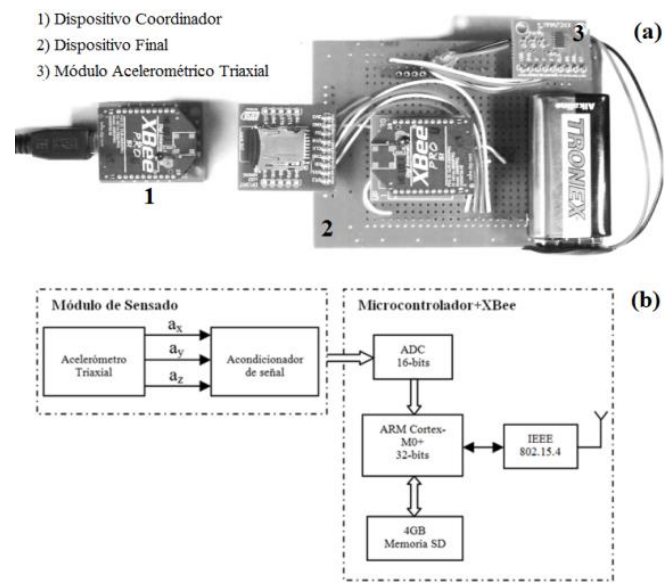

Fig. 1. Hardware implementado. a) Fotografía del Hardware y b) Esquema del conjunto dispositivo final y módulo de sensado. Fuente: Autores

Dentro de las bondades de la plataforma Freescale Freedom para la implementación del nodo se destaca la excelente resolución de la conversión (siendo $50 \mathrm{uV}$ el valor más pequeño de voltaje que logra resolver), que para efectos del análisis de las vibraciones mecánicas en el dominio del tiempo (Scott et al., 2001) es adecuada.

\subsection{Diseño de los Experimentos}

En la Fig. 2 se muestra un esquema de la zona de pruebas, en la cual se encuentra dos edificios, donde se implementó la RSAI. El nodo coordinador se ubica en el extremo de un edificio y el nodo sensor se desplaza a una distancia ajustable entre $50 \mathrm{~m}$ y $200 \mathrm{~m}$ respecto al coordinador. Entre ambos equipos se interpone una edificación con elementos esenciales como ventanas, puertas, columnas y muros. El coordinador fue ubicado a una altura de $2 \mathrm{~m}$ respecto a la superficie del suelo, y el nodo sensor se encuentra a 0 
m del suelo, justo en el punto de medición de las señales acelerométricas.

En la zona donde se ejecutaron las pruebas se encuentra un terreno adecuado para estudios de las propiedades físicas de las capas superficiales donde se ubicó un actuador mecánico (tipo martillo percutor). Este impacta el suelo generando ondas elásticas, las cuales son registradas por el nodo sensor para ser enviadas posteriormente al coordinador (sitio de análisis).

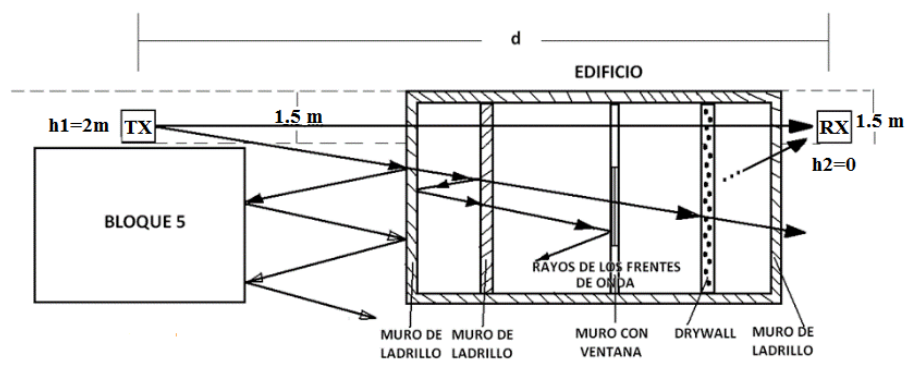

Fig. 2. Diagrama de bloques (vista en planta) de la ubicación de los obstáculos y los módulos sensor (TX) y coordinador (RX), enseñando las posibles direcciones de los rayos de los frentes de onda. La distancia d en la figura corresponde a $150 \mathrm{~m}$.

Fuente: Autores

El estudio experimental realizado consta básicamente de dos etapas. La primera es diseñada para evaluar la calidad del enlace, en la cual se realizó la medición del número de paquetes exitosos recibidos por el coordinador en función de la distancia entre los nodos. El conjunto de datos se obtuvo al inyectar una perturbación mecánica al suelo bajo prueba, a continuación se midió las aceleraciones con el nodo sensor y su transmisión al coordinador para finalmente establecer la razón entre el número de paquetes enviados y recibidos de forma exitosa.

En la segunda, se determinó las máximas frecuencias de muestreo a diferentes velocidades de transmisión y bajo dos configuraciones diferentes de operación: i) El SAI captura, hace la conversión A/D de las vibraciones y transmite la información simultáneamente al coordinador (on-line); ii) El SAI captura y hace la conversión A/D de las vibraciones, guarda estos datos en una memoria no volátil, y al finalizar el tiempo de sensado se transmite toda la información al coordinador (off-line). Para medir los tiem- 
pos efectivos de conversión y emisión de datos, se utilizaron los módulos temporizadores presentes en los nodos sensor y coordinador, y la información capturada se recolectó en tablas. La finalidad de esta etapa es verificar la capacidad de la red inalámbrica de capturar adecuadamente las señales de las aceleraciones del suelo, bajo la luz del teorema del muestreo de Nyquist-Shannon.

\section{RESULTADOS DE LA PRUEBA DE CAMPO Y DISCUSIÓN}

En la primera etapa experimental, para examinar la incidencia de la distancia coordinador-sensor y de la presencia de obstáculos, se tomaron tres distancias de referencia: $50 \mathrm{~m}, 100 \mathrm{~m}$ y $200 \mathrm{~m}$. Posteriormente, se determinó la razón de la cantidad de paquetes enviados y recibidos por los nodos para diferentes velocidades de transmisión. Los resultados de la prueba se consignan en la Tabla 1.

Tabla 1. Paquetes transmitidos satisfactoriamente al coordinador. Las distancias coordinador-dispositivo final fijadas en la prueba son $50 \mathrm{~m}, 100 \mathrm{~m}$ y $200 \mathrm{~m}$, y están dispuestas para cada velocidad de transmisión en el orden de arriba hacia abajo, respectivamente. Fuente: Autores

\begin{tabular}{ccc}
\hline $\begin{array}{c}\text { Velocidad } \\
\text { (bps) }\end{array}$ & $\begin{array}{c}\text { Tiempo de } \\
\text { transmisión (us) }\end{array}$ & $\begin{array}{c}\text { \% Paquetes } \\
\text { Entregados }\end{array}$ \\
\hline \multirow{3}{*}{115200} & 925 & 100 \\
& 930 & 100 \\
n/a & 28 \\
\hline \multirow{2}{*}{19200} & 5265 & 100 \\
& 5281 & 100 \\
& n/a & 30 \\
\hline \multirow{3}{*}{4800} & 20941 & 100 \\
& 21019 & 100 \\
& n/a & 30 \\
\hline
\end{tabular}

Estos resultados muestran que existe una disminución notable de la confiabilidad de los datos recibidos en [\% de paquetes entregados] para longitudes del enlace inalámbrico igual o superior a los $200 \mathrm{~m}$, bajo la presencia de un obstáculo físico entre los módu- 
los (edificio); y una baja correlación entre la confiabilidad y la velocidad de transferencia de los datos.

La pérdida de paquetes entre los nodos cuando la distancia es igual o superior a los $200 \mathrm{~m}$, es producida por la atenuación generada por los obstáculos durante la radio-propagación sin línea de vista (NLOS). En este caso los frentes de ondas al interactuar con los obstáculos sufren difracciones y reflexiones múltiples, lo cual podría dar lugar al caso crítico donde las ondas que alcanzan a llegar al receptor interfieren entre sí destructivamente, incrementando las pérdidas en el canal aparte de las absorciones propias del medio de propagación (aire). Keenan \& Motley (1990) desarrollaron un modelo empírico (MK) que sirve para evaluar la atenuación del canal para enlaces NLOS, que incluye el efecto de los muros y suelos de las edificaciones, útil en el intervalo de UHF (Ultra High Frequency) y Microondas. Se describe de forma aproximada la atenuación del enlace bajo dicho modelo mediante (1):

$L_{M K}=L_{F S}+\sum_{i=1}^{M} K W_{i} L_{W}+\sum_{i=1}^{M} K F_{i} L_{F}+L_{C}$

donde $L_{M K}(d B)$ es la atenuación del canal NLOS propuesta por Keenan y Motley (1990); $L_{F S}(d B)$ es la atenuación del canal en espacio libre; $K W_{i}$ es el número de muros presentes en la edificación; $K W_{i}$ es el número de muros presentes en la edificación; $K F_{i}$ el número de suelos donde la onda se refleja; $L_{W}(d B)$ es la atenuación introducida por el muro i-ésimo (Keenan \& Motley (1990) proponen valores experimentales para este parámetro dependiendo de los materiales de fabricación y el espesor de los muros); $L_{C}(d B)$ es la atenuación empírica que sirve para corregir el modelo MK, bajo las diferentes configuraciones de los obstáculos en el interior de las edificaciones. La expresión que rige a $L_{F S}$ está dada por (2):

$L_{F s}=10 \log \left[\frac{\lambda^{2}}{(4 \pi d)^{2}}\right]$

con $\lambda(m)$ como la longitud de onda y $d(m)$ la distancia entre la estación transmisora y la estación receptora. 
La potencia del receptor (en $\mathrm{dBm}$ ) se calcula teniendo presente la potencia media del transmisor, la ganancia de las antenas, la atenuación de los cables empleados para conectar las antenas, y las pérdidas de propagación NLOS predichas por el modelo MK, tal como se ilustra en (3).

$P_{R X}=G_{\text {AntenaTX }}-L_{\text {CableTX }}-L_{M K}+G_{\text {AntenaRX }}-L_{\text {CableRX }}+P_{T X}$

donde $P_{R X}(\mathrm{dBm})$ es la potencia promedio en la entrada del receptor; $P_{T X}(\mathrm{dBm})$ es la potencia promedio inyectada a la antena del transmisor; $\left\{G_{\text {AntenaTX }}, G_{\text {AntenaRX }}\right\}$ (dBi) son las ganancias de potencia isotrópica de las antenas y $\left\{L_{\text {CableTX }}, L_{\text {CablerX }}\right\}(d B)$ son las atenuaciones de los cables que conectan las antenas. Las antenas empleadas en los módulos inalámbricos son monopolos de $\lambda / 4$ para $2.4 \mathrm{GHz}$, con conectores on-board tipo UFL que no requieren de cables de conexión, y con ganancias cada una de $1.5 \mathrm{dBi}$.

En base a la Fig. 2, teniendo en cuenta que la distancia d es de $150 \mathrm{~m}$, se listan los materiales de los obstáculos, y luego se procede a consultar tablas experimentales que indexan la atenuación que generan estos elementos en función del tipo de material y la longitud de onda empleada (Keenan \& Motley, 1990). A partir de lo consultado se obtuvieron los siguientes parámetros: $L_{W 1}=6.9 \mathrm{~dB}$ (Muros de ladrillo y hormigón de $30 \mathrm{~cm}$ ), $L_{W 2}=3.4 \mathrm{~dB}$ (muro con ventana o drywall), y $L_{F}=18.3 d B$ (Baldosas convencionales). Con estos valores fue posible estimar la atenuación del canal en función de la distancia existente entre los módulos inalámbricos. Si la potencia promedio emitida por el módulo sensor es igual a $20 \mathrm{dBm}$, entonces la potencia en la entrada del receptor será igual a $P_{R X}=-113.55-20 \log (d)$, con $d$ en $\mathrm{km}$. La potencia umbral del módulo coordinador es de $-100 \mathrm{dBm}$, lo cual implica que la distancia máxima permisible para el radioenlace exitoso es aproximadamente $210 \mathrm{~m}$, consistente con los resultados obtenidos experimentalmente.

Consecuentemente, los resultados de la segunda etapa, donde se verifica la capacidad de la RSAI de capturar adecuadamente las señales del suelo, son mostrados en la Tabla 2 donde se ilustran los tiempos de conversión medidos para diferentes velocidades de transmisión, empleando el módulo temporizador del sensor, usan- 
do la configuración i (on-line), tal como se describió en el diseño de la etapa 2.

Tabla 2. Tiempo total empleado para realizar la conversión A/D de las señales ax, ay y az y su transmisión al Coordinador en la configuración on-line. Fuente: Autores

\begin{tabular}{ccccccc}
\hline Velocidad $(\mathrm{bps})$ & 115200 & 57600 & 38400 & 19200 & 9600 & 4800 \\
\hline Tiempo $(\mu \mathrm{s})$ & 930 & 1800 & 2670 & 5290 & 10500 & 21000 \\
\hline
\end{tabular}

A partir de estos resultados, se concluye que no es factible a la luz del teorema del muestreo de Nyquist, utilizar velocidades inferiores a 115200 bps para capturar señales análogas con frecuencias superiores a los $500 \mathrm{~Hz}$. Alternativamente, usando la configuración ii (off-line), se estimó el tiempo empleado por el nodo para realizar el proceso de conversión A/D y la escritura de los datos en una memoria no volátil de tecnología Flash, donde el resultado obtenido fue de 160 us. Con esta configuración se registran una frecuencia de muestreo de $6250 \mathrm{~Hz}$, lo suficientemente alta para capturar señales con frecuencias máximas de hasta 3 $\mathrm{kHz}$ aproximadamente.

La exploración sísmica aplicada en la detección de objetos enterrados a baja profundidad empleando ondas acústicas, cimentada por autores como Donskoy (2008), Schröder y Scott (2006) requiere de sistemas de adquisición de datos con frecuencias de muestreo adecuadas para capturar señales análogas con frecuencias máximas de hasta $1 \mathrm{kHz}$ aproximadamente, y la suficiente capacidad de memoria para almacenar entre $1 \mathrm{~s}$ y $10 \mathrm{~s}$ de estas señales con una resolución aceptable (entre 12 y 16 bits). Bajo los requerimientos técnicos mencionados, se pudo verificar a partir de los espectros de magnitud y de las gráficas en el tiempo de las muestras de las aceleraciones medidas en la etapa 2 (ver Fig. 3), que los anchos de bandas de las señales son aproximadamente iguales a $300 \mathrm{~Hz}$, y son consecuentes con los estimados por algunos autores (Donskoy, 2008; Schröder \& Scott, 2006); al igual que las respuestas impulsivas en el tiempo que se ajustan a las generadas por fuentes puntuales de energía mecánica. Estos resultados validan la calidad de las muestras capturadas en el experimento. 
(a)

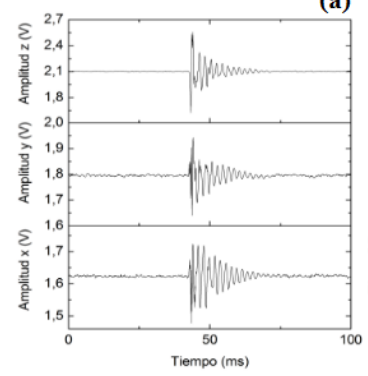

.

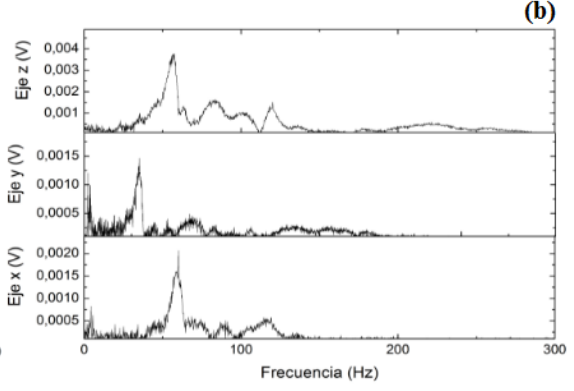

Fig. 3. Recepción y procesamiento de datos. a) Señales recibidas en el coordinador correspondientes a la aceleración del SAI acoplado al terreno de estudio. b) Transformada de Fourier (magnitud pico) de las señales acelerométricas.

Fuente: Autores

Para implementar una RSAI con una mayor cantidad de nodos es absolutamente importante tener en mente la longitud del enlace entre los módulos inalámbricos, la cual está dominada fuertemente por los obstáculos presentes en la primera zona de Fresnel, en ese sentido se podría incrementar a partir del uso de enrutadores. Adicionalmente, una estrategia válida para aumentar la vida útil de la batería del dispositivo como lo sugiere (Chebrolu et al., 2008), consistiría en colocar los dispositivos finales en modo sleep la mayor parte del tiempo y modificar este estado únicamente cuando se presente el evento de interés. Finalmente, cabe mencionar que la transferencia de datos a una baja tasa de transmisión no es un factor crítico en algunas aplicaciones, pero si es fundamental garantizar que la cantidad de datos recolectados (un conjunto usualmente voluminoso) posea un tamaño adecuado que permita caracterizar el patrón de vibraciones para las aplicaciones mencionadas.

\section{CONCLUSIONES}

Llevar a cabo el registro de señales de vibraciones mecánicas en suelos con las exigencias que impone su prospección a partir de la inyección de ondas mecánicas, y transmitirlas en tiempo real con módulos XBee en una configuración on-line, es una tarea inviable debido a que las velocidades de transmisión características 
de los módulos no pueden responder a la tasa de muestreo necesaria para representar adecuadamente la señal. Una alternativa para superar esta limitante y explotar las bondades de bajo consumo que ofrece esta tecnología, es emplear una configuración offline, donde se captura la señal y se almacena en un buffer (una memoria no volátil), y una vez finalizada la medición, se puede transmitir. Adicionalmente, la tecnología inalámbrica empleada, es fuertemente susceptible a la presencia de obstáculos físicos lo cual se podría mejorar empleando dispositivos enrutadores que permitan incrementar la longitud de enlace.

\section{AGRADECIMIENTOS}

Los autores agradecen a la Institución Universitaria Pascual Bravo, la Universidad Santo Tomás y a la Universidad Cooperativa de Colombia por brindar los espacios y financiación para llevar a cabo este trabajo. A Sebastián Villa por su lectura, revisión crítica y sugerencias a esta contribución.

\section{REFERENCIAS}

Ashok, N., Patel,Y. (2012). Zigbee: A Low Power Wireless Technology For Industrial Applications. International Journal of Control Theory and Computer Modelling (IJCTCM), 2 (3), 27-33

Chebrolu, K., Raman, B., Mishra, N., Valiveti, P. K., Kumar, R., (2008). BriMon: A Sensor Network System for Railway Bridge Monitoring. Proc. 6th International Conference on Mobile Systems, Applications, and Services (MobiSys), 2-16.

Elagib, I., Artoli, A.M., Habbani, F., Badawi, M., (2013).Monte Carlo simulation of pu-be, am-be and cf-252 neutrons backscattering from buried explosives in dry soil. IEEE 2013 International Conference on Computer Applications Technology (ICCAT), 1-4.

García-García, A., Levey, M. D., Watsond, E. B., (2013). High resolution seismic study of the Holocene infill of the Elkhorn Slough, central California. Continental Shelf Research, 55, 108-118.

Ghariani, N., Chaoui, M., Ghariani, H., Lahiani, M., (2011). Design of a Digital Communication System Based on a XBee Module for Biomedi- 
cal Applications. 8th International Multi-Conference on Systems, Signals \& Devices, 11, 1-5.

Huang H., Carande, B., Tang, R., Oiler, J., Dmitriy, Z., Vadim, A., Yu, H., (2013). Development of a micro seismometer based on molecular electronic transducer technology for planetary exploration. IEEE 26th International Conference on Micro Electro Mechanical Systems (ICMEMS), 629-632.

Karpat, E., (2013). Subsurface Imaging Analysis for Multiple Objects.IEEE 2013 4th International Conference on Intelligent Systems, Modelling and Simulation (ICISMS), 195-198.

Keenan, J.M, Motley, A.J, (1990). Radio coverage in buildings. British Telecom, Technology journal, vol. 8.

Kim, S., Pakzad, S., Culler, D., Demmel, J., Fenves, G., Glaser, S., Turon, M., (2007). Health Monitoring of Civil Infrastructures using Wireless Sensor Networks. IPSN '07 Proceedings of the 6th international conference on Information processing in sensor networks, 254-263.

Lou J., Jin T., Liang, F.,Zhou Z., (2013). A Novel Prescreening Method for Land-Mine Detection in UWB SAR Based on Feature Point Matching. IEEE Communications Magazine, IEEE Transactions on Geoscience and Remote Sensing, 51(6), 3706-3714.

Owojaiye, G., Sun, Y., (2013). Focal design issues affecting the deployment of wireless sensor networks for pipeline monitoring. Ad Hoc Networks, 11(3), 1237-1253.

Pérez-Gracia, V., Caselles, J. O., Clapés, J., Martinez, G., Osorio, R., (2013). Non-destructive analysis in cultural heritage buildings: Evaluating the Mallorca cathedral supporting structures. NDT \& E International, 59, 40-47.

Picozzi, M., Milkereit, C., Parolai, S., Jaeckel, K.-H., Veit, I., Fischer, J., Zschau, J., (2010). GFZ Wireless Seismic Array (GFZ-WISE), a Wireless Mesh Network of Seismic Sensors: New Perspectives for Seismic Noise Array Investigations and Site Monitoring. IEEE Sensors, 10(4), 3280-3304.

Ryden, N., Mooney, M. A., (2009). Analysis of surface waves from the lightweight deflectometer. Soil Dynamics and Earthquake Engineering, 29, 1134-1142.

Savazzi, S., Spagnolini, U., Goratti, L., Molteni, D. (2013). Ultra-wide band sensor networks in oil and gas explorations. IEEE Communications Magazine, 51(4), 150-160.

Schröder, C. T., Scott, W. R., (2000). A Finite-Difference Model to Study the Elastic-Wave Interactions with Buried Land Mines. IEEE Transactions on Geoscience and Remote Sensing, 38(4), 1505-1512. 
Scott, W. R., Martin, J. S., Larson, G. D., (2001). Experimental Model for a Seismic Landmine Detection System. IEEE Transactions on Geoscience and Remote Sensing, 39(6), 1155-1164.

Shu, S., Xiong, J., Ma, C., Tang, Y., (2012). A small-scale wireless transmission network attached to large-scale seismic acquisition and recording system. IEEE International Conference on Automatic Control and Artificial Intelligence (ACAI 2012), 292-295.

Steeples, D., (2005). Near-surface geophysics: 75 years of progress in the Leading Edge. Society of Exploration Geophysics, 24, 582-585.

Sun, J., Wenb, J., (2013). Target location method for pipeline pre-warning system based on HHT and time difference of arrival. Measurement, 46(8), 2716-2725.

Trebi-Ollennu, A., Rankin, A.L., Yang C., Tso, K.S., Deen, R.G., Aghazarian, H., Kulczycki, E.A., Bonitz, R.G., Alkalai, L., (2013). Instrument deployment testbed: For planetary surface geophysical exploration. IEEE 2013 Aerospace Conference, 1-14.

Villa D., Moya F., López J., (2009). Infraestructura para la integración de redes de sensores y actuadores en entornos inteligentes. Disertación Doctoral, Universidad de Castilla-La Mancha, España.

Virieux, J., (1986). P-SV wave propagation in heterogenous media: Velocity stress finite-difference method. Geophysics, 51, 889-901.

Watts, P.E., (2013). System reliability for seismic sensing. IEEE 2013 Proceedings-Annual Reliability and Maintainability Symposium (RAMS), 1-4.

Xiang, N., Sabatier, J. M., (2000). Land mine detection measurements using acoustic-to-seismic coupling. Proceedings of SPIE, 4038, 645655.

Xu, N., Rangwala, S., Chintalapudi, K. K., Ganesan, D., Broad, A., Govindan, R., Estrin, D., (2004). A Wireless Sensor Network for Structural Monitoring. Proc. 2nd International Conference on Embedded Networked Sensor Systems (SenSys), 13-24.

Zhao, W., Forte, E., Pipan, M., Tian, G., (2013). Ground Penetrating Radar (GPR) attribute analysis for archaeological prospection. Journal of Applied Geophysics, 97, 107-117. 